\title{
Nocardia asteroides
}

National Cancer Institute

\section{Source}

National Cancer Institute. Nocardia asteroides. NCI Thesaurus. Code C86611.

A species of aerobic, Gram-positive, rod shaped bacteria assigned to the phylum Actinobacteria. This species is catalase and urease positive, acid-fast, hydrolyzes esculin but not casein, reduces nitrate, and characteristically produces a mycelium that fragments into bacillary and coccoid elements. N. asteroides is naturally found in soil, ans lake and marine sediment where it digests plant matter; but in humans it is an opportunistic pathogen and one of the causative agents of nocardiosis in immunocompromised individuals. 